\title{
Effects of Irradiation on Epithelial Wound Healing and Microbial Diversity in an in-vitro Oral Mucosa Model
}

\author{
Tine De Ryck ${ }^{1}$, Tom Boterberg ${ }^{2}$, Frederiek-Maarten Kerckhof ${ }^{3}$, Joachim De Schrijver ${ }^{4}$, Marc Bracke ${ }^{1}$, Tom Van de Wiele ${ }^{3^{*}}$ and Barbara Vanhoecke ${ }^{3}$ \\ ${ }^{1}$ Laboratory of Experimental Cancer Research (LECR), Ghent University, 9000 Ghent, Belgium \\ ${ }^{2}$ Department of Radiation Oncology, Ghent University Hospital, 9000 Ghent, Belgium \\ ${ }^{3}$ Laboratory of Microbial Ecology and Technology (LabMET), Ghent University, 9000 Ghent, Belgium \\ ${ }^{4}$ Laboratory of Bioinformatics and Computational Genomics (Biobix), Ghent University, 9000 Ghent, Belgium
}

*Corresponding author: Tom Van de Wiele, Laboratory of Microbial Ecology and Technology (LabMET), Coupure Links 653 Building A, 9000 Ghent, Belgium, Tel: +32 926459 12; Fax: +32 926462 48; E-mail: tom.vandewiele@ugent.be

Received date: Feb 09, 2015, Accepted date: Mar 16, 2015, Publication date: Mar 19, 2015

Copyright: (c) 2015 Ryck TD, et al. This is an open-access article distributed under the terms of the Creative Commons Attribution License, which permits unrestricted use, distribution, and reproduction in any medium, provided the original author and source are credited.

\begin{abstract}
The impact of irradiation on host-microbe crosstalk is still underexplored. By use of an in vitro oral mucosa model, we show an impact of irradiation on epithelial wound healing depending on the microbial composition and functionality. 454-pyrosequencing analyses pointed to a slight increase in abundance of Rothia, Granulicatella and Gemella in our model after irradiation. Further research is needed to unravel the effects of irradiation on the oral microbiota and the host-microbe interactions more in detail.
\end{abstract}

Keywords: Host-microbe; Irradiation; 454-pyrosequencing; In vitro oral mucosa model; Wound healing

\section{Abbreviations:}

PBS: Phosphate Buffered Saline; BHI: Brain Heart Infusion; CFU: Colony Forming Units; PCR-DGGE: Polymerase Chain reactionDenaturing Gradient Gel Electrophoresis

\section{Introduction}

Oral mucositis is an important side effect of cancer therapies. In $91 \%$ of the patients with head and neck cancer, radiotherapy results in the development of mucositis [1]. The pathobiological process of mucositis can be subdivided in 5 phases: the initiation phase, the primary damage response followed by signal amplification, the ulceration and the healing phase [2,3]. From these, the healing phase is highly variable between patients and is probably the least understood $[2,4]$. Although differences in treatment schedules, irradiation doses and individual radiation sensitivity may be responsible for the differences in wound healing, the composition of the oral microbiome is also thought to be one of the determining factors. An oral core microbiome has been described for healthy individuals with Streptococcus being a predominant genus; yet, genera like Neisseria, Prevotella, Veillonella or Haemophilus can also become highly abundant. At species level, this interindividual variation is even larger [5]. A recent review describes important shifts in the core oral microbiome due to radiotherapy, with an increased abundance of Gram-negative species and Lactobacillus spp. [6]. Irradiation has thus a clear impact on the host and on the oral microbiome, however it is unknown to what extent irradiation affects the host-microbe crosstalk, particularly during the wound healing phase of mucositis. Here, we used an in vitro oral mucosa model [7] to study the impact of oral microbes on the dynamics of the wound healing process following irradiation more in detail.

\section{Materials and Methods}

\section{Epithelial cell line}

TR146 cells, an oral squamous carcinoma cell line derived from a local lymph node metastasis was obtained from Clare Hall Laboratories (Cancer Research UK). The Dulbecco's modified Eagle's cell culture Medium (DMEM) (Gibco, Belgium) was supplemented with $10 \%$ heat-inactivated fetal bovine serum (Greiner bio-one, Belgium), $22.8 \mu \mathrm{g} / \mathrm{mL}$ penicillin-streptomycin (5000 U/mL; Gibco, Belgium) and $2.5 \mu \mathrm{g} / \mathrm{mL}$ amphotericin B (Bristol-MyersSquibb, Belgium). Cells were cultured at $37^{\circ} \mathrm{C}$ and $10 \% \mathrm{CO}_{2}$ and regularly controlled to be mycoplasma free (MycoAlert Mycoplasma Detection kit; Lonza, USA).

\section{Irradiation}

A single $6 \mathrm{MV}$ photon beam was generated using a linear accelerator (SLi-18, Elekta, UK). The 24-well plates were placed on top of a $1.5 \mathrm{~cm}$ Perspex (polymethylmethacrylate) plate to compensate for the build-up effect and irradiated from below. The model was irradiated with a total dose of $10 \mathrm{~Gy}$ at an instantaneous dose rate of $430 \mathrm{cGy} / \mathrm{min}$.

\section{Oral swab collection}

Before sampling, the oral cavity of a healthy volunteer was extensively flushed with drinking water. A sterile cotton swab was used to rub the buccal mucosa 10 times before submersion in $2 \mathrm{~mL}$ of synthetic saliva buffer. Briefly, an organic buffer ( $500 \mathrm{mg} / \mathrm{L}$ ureum), an inorganic buffer $\left(2.987 \mathrm{~g} / \mathrm{L} \mathrm{KCl}, 2.96 \mathrm{~g} / \mathrm{L} \mathrm{NaH}_{2} \mathrm{PO}_{4}, 0.667 \mathrm{~g} / \mathrm{L} \mathrm{KSCN}\right.$, $1.9 \mathrm{~g} / \mathrm{L} \mathrm{Na}_{2} \mathrm{SO}_{4}, 0.993 \mathrm{~g} / \mathrm{L} \mathrm{NaCl}$ and $\left.10.8 \mathrm{mM} \mathrm{NaOH}\right)$ and an additive buffer (483 mg/L amylase, $167 \mathrm{mg} / \mathrm{L}$ mucin and $50 \mathrm{mg} / \mathrm{L}$ uric acid) were autoclaved and mixed (4:3:3) immediately before use. After incubating the oral swabs for $15 \mathrm{~min}$ at $37^{\circ} \mathrm{C}$, the microbial suspensions were snap frozen in liquid nitrogen and stored at $-80^{\circ} \mathrm{C}$ 
until the experiments were performed. For all experiments, a different oral swab was taken from the same healthy volunteer.

\section{Oral mucosa model}

To co-culture microbiota and TR146 epithelial cells in noninfectious conditions, we used our recently published oral mucosa model [7]. Briefly, $75 \mu \mathrm{L}$ of an agar/mucin solution was spotted on the porous membrane (pore size $0.4 \mu \mathrm{m}$ ) of a 24 -well plate Transwell ${ }^{\circ}$ system (Corning inc., USA) and allowed to solidify for at least $30 \mathrm{~min}$. After thawing at room temperature, $20 \mu \mathrm{L}$ of the microbial samples ( 3.2 log/filter) were brought on top of the agar/mucin layer. In the basal compartment, a confluent layer of TR146 cells was generated by seeding 300000 TR146 cells into a well after labelling with DiI cell labelling solution (Life technologies Europe, Belgium). Cells were allowed to attach and grow for 3 days prior to wounding.

\section{Wound healing assay}

TR146 monolayers were scratched using a sterile plastic pipette tip. Floating cells were removed by replacing the medium with fresh serum-free, antibiotics-free DMEM. Filters containing the microbiota and the agar/mucin layer were placed on top of the wounded cells prior to irradiation. By means of an automated fluorescence microscope (Zeiss Axiovert 200M), pictures of 4 selected points along the wounds in each well were taken immediately after irradiation $(0 \mathrm{~h})$ and $24 \mathrm{~h}$ after incubation at $37^{\circ} \mathrm{C}$ and $5 \% \mathrm{CO}_{2}$. Migration of the cells into the wounded area in the presence and absence of oral microbiota was assessed by comparing the micrographs after 0 and $24 \mathrm{~h}$ (Figures $1 \mathrm{~A}$ and $2 \mathrm{~A}$ ). The relative surface (\%) of the wound was determined by dividing the wound surface after $24 \mathrm{~h}$ by the wound surface at time 0 . For this we made use of a custom-made macro in ImageJ [7]. For each well, the mean value of the wound surface was calculated $(n=1)$ and used for further analysis. In total, 5 independent experiments were performed, each with 2 to 4 wells per condition.

\section{Microbial enumeration}

To enumerate the microbiota at the start of the experiment, 10-fold dilutions of the oral swab suspension were prepared in sterile PBS and plated on BHI agar plates (Sigma, Belgium) using the microdilution plating technique.

After $24 \mathrm{~h}$ of co-culture, microbiota in the apical compartment were suspended in $200 \mu \mathrm{L}$ of sterile PBS before plating 10-fold dilutions as described above.

\section{4 pyrosequencing}

DNA extraction and 454 pyrosequencing analysis of the apical compartment was performed as described earlier [7]. On average, 12 907 number of reads were generated by pyrosequencing for each sample. After processing the results, operational taxonomic units were classified using the RDP database.

\section{Statistics}

Statistical analyses were performed with SPSS statistics 22. After normality check with the Shapiro-Wilk assay, an ANOVA, Student's T, Kruskal Wallis or Mann Whitney-U test was used. Bonferroni correction was performed to correct for multiple comparisons.
All other techniques used for this study are described in detail in De Ryck et al. [7].

\section{Results and Discussion}

In patients suffering from oral mucositis, the healing phase spontaneously starts 2-3 weeks after completion of radiotherapy. Signals originating from the extracellular matrix promote wound healing by stimulating migration, proliferation and differentiation of the epithelium [2]. We previously showed our in vitro oral mucosa model to be useful for the study of effects of oral microbiota on the wound healing capacity of different oral-derived cell lines [7]. In nonirradiated conditions, epithelial recovery was significantly reduced with $12-16 \%$ in the presence of microbiota (Figures $1 \mathrm{~B}$ and $2 \mathrm{~B}, 0 \mathrm{GY}$; $\mathrm{p}<0.001$ ). After irradiation however, our experimental data could be categorized in 2 different sets.

In 3 of the 5 independent experiments (set 1) the inhibitory effect of the microbiota on wound healing was more pronounced after irradiation as compared to the non-irradiated condition (Figure 1B, $10 \mathrm{~Gy} ; \mathrm{p}<0.001)$. In contrast, no effect of the microbiota after irradiation could be noticed in the other 2 independent experiments (set 2; Figure $2 \mathrm{~B}, 10 \mathrm{~Gy} ; \mathrm{p}=1$ ). Interestingly, plate counts revealed a lower number of microbiota in set 2 after $24 \mathrm{~h}$ of co-culture compared to set $1(\mathrm{p}=0.053)$. We hypothesized that this might be the result of a difference in microbial load and/or composition in the oral cavity of the healthy volunteer at the time of sampling. While the difference in microbial concentrations between the 2 experimental datasets was not influenced by irradiation (Table 1; $\mathrm{p}_{\text {set1:0Gy-10Gy }}=0.979$; $\mathrm{p}_{\text {set2:0Gy-10Gy }}=0.800$ ), our data suggest that microbial growth is an important determining factor for the wound healing process after irradiation.

\begin{tabular}{|l|l|l|}
\hline $\begin{array}{l}\text { Microbial Counts (log } \\
\text { CFU/Filter) }\end{array}$ & $\mathbf{0}$ G & $10 \mathrm{~Gy}$ \\
\hline Set 1 & $7.29 \pm 0.51$ & $7.31 \pm 0.74$ \\
\hline Set 2 & $5.88 \pm 1.55$ & $5.77 \pm 1.08$ \\
\hline
\end{tabular}

Table 1: Microbial enumeration of the apical compartment after $24 \mathrm{~h}$ of co-culture with TR146 cells in irradiated (10 Gy) or non-irradiated (0 Gy) conditions (mean $\pm \mathrm{SD}$ ).

Irradiation has previously been shown to prolong the lag phase for foodborne pathogens delaying the stationary phase [8]. As a consequence, microbial metabolites might be present at concentrations that are too low to have an effect on the epithelial cells. A microbial metabolite that was present in our in vitro model was Dlactic acid [7]. Here we show that oral microbiota increase the levels of D-lactic acid after $24 \mathrm{~h}$ in both datasets (Figures $1 \mathrm{C}$ and 2C; set1: $\mathrm{p}_{0 \mathrm{~Gy}}<0.001$ and $\mathrm{p}_{10 \mathrm{G}} \mathrm{y}<0.001$; set2: $\mathrm{p}_{0 \mathrm{~Gy}}=0.167$ and $\left.\mathrm{p}_{10 \mathrm{G}} \mathrm{y}=0.445\right)$, but that irradiation did not have an effect on this increase $\left(\mathrm{p}_{\text {set } 1}=0.308\right.$; $\mathrm{p}_{\text {set2 }}=1$ ). Therefore, D-lactic acid present in the co-culture medium could not explain the difference in effect on wound healing of both sets. Furthermore, the epithelial wound healing was not affected in presence of exogenous D-lactic acid up to $5000 \mu \mathrm{g} / \mathrm{mL}$ (data not shown). 
Citation: Ryck TD, Boterberg T, Kerckhof FM, Schrijver JD, Bracke M, et al. (2015) Effects of Irradiation on Epithelial Wound Healing and Microbial Diversity in an in-vitro Oral Mucosa Model. J Nucl Med Radiat Ther 6: 218. doi:10.4172/2155-9619.1000218

Page 3 of 4

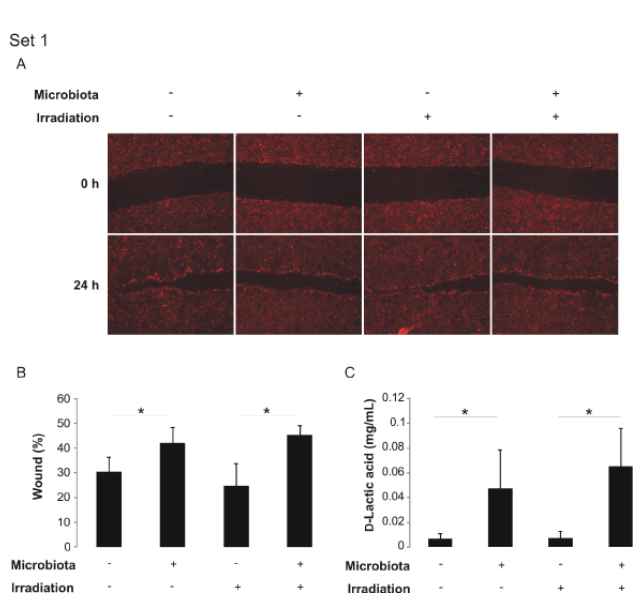

Figure 1: Effects of irradiation on microbe-epithelial interactions in the first set of observations. A: Representative examples of the micrographs of the wound healing capacity of TR146 cells at $0 \mathrm{~h}$ and $24 \mathrm{~h}$ in the absence or presence of oral microbiota, both in irradiated (10 Gy) or non-irradiated (0 Gy) conditions B: Wound healing capacity of TR146 cells in the absence or presence of oral microbiota (\% of open wound after $24 \mathrm{~h}$, mean $+\mathrm{SD} ;{ }^{*} \mathrm{p}<0.05$ ), both in irradiated (10 Gy) or non-irradiated (0 Gy) conditions. C: Concentrations of D-lactate in the co-culture medium in the absence or presence of oral microbiota, both in irradiated (10 Gy) or non-irradiated $(0 \mathrm{~Gy})$ conditions (mean $\left.+\mathrm{SD} ;{ }^{*} \mathrm{p}<0.05\right)$.

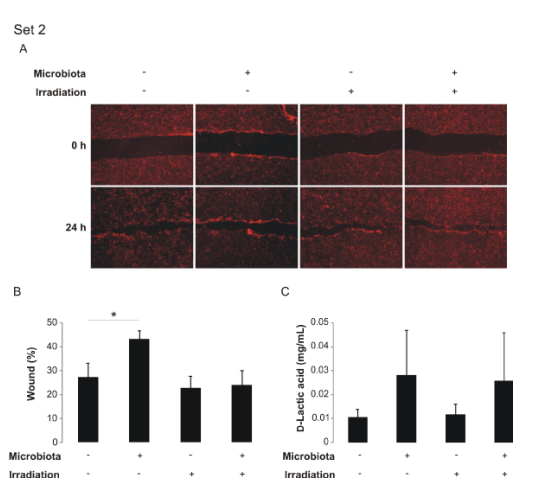

Figure 2: Effects of irradiation on microbe-epithelial interactions in the second set of observations. A: Representative examples of the micrographs of the wound healing capacity of TR146 cells at $0 \mathrm{~h}$ and $24 \mathrm{~h}$ in the absence or presence of oral microbiota, both in irradiated (10 Gy) or non-irradiated (0 Gy) conditions B: Wound healing capacity of TR146 cells in the absence or presence of oral microbiota (\% of open wound after $24 \mathrm{~h}$, mean $+\mathrm{SD}$; ${ }^{*} \mathrm{p}<0.05$ ), both in irradiated (10 Gy) or non-irradiated (0 Gy) conditions. C: Concentrations of D-lactate in the co-culture medium in the absence or presence of oral microbiota, both in irradiated (10 Gy) or non-irradiated (0 Gy) conditions (mean $\left.+\mathrm{SD} ;{ }^{*} \mathrm{p}<0.05\right)$.

Next, we evaluated to what extent irradiation induces shifts in the microbial composition of the oral biofilm after $24 \mathrm{~h}$ in our in vitro oral mucosa model. To this end, PCR-DGGE was used as a quick screening technique. Although shifts in microbial composition were noted upon irradiation, this was only observed in $50 \%$ of the experiments (Figure $3 \mathrm{~A})$. In all samples, an identical GC-poor group of microbiota could be detected (arrow-marked on Figure 3A), most probably representing the oral core microbiome.

454 pyrosequencing analysis of the microbiota co-incubated with an epithelial monolayer for 24 or $48 \mathrm{~h}$, showed that irradiation induces a small increase $(<1 \%)$ in Rothia, Granulicatella and Gemella species (Figure $\quad 3 B ; \quad p_{\text {Rothia-24h }}=0.196, \quad p_{\text {Granulicatella- } 24 \mathrm{~h}}=0.062$,

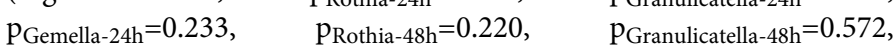
$\left.\mathrm{p}_{\text {Gemella-48h }}=0.207\right)$. Unlike Granulicatella and Gemella that are omnipresent in the oral cavity, Rothia is mostly linked with tooth surfaces [9]. Interestingly, Rothia species were reported to become more abundant in head and neck cancer patients during radiotherapy, whereas Granulicatella species were shown to decrease after irradiation $[10,11]$. Therefore Granulicatella species are not likely to be the causing agents of post-radiation diseases [11]. In contrast, different cases of Rothia bacteraemia have been reported of which $36 \%$ could be linked with oral mucositis [12].
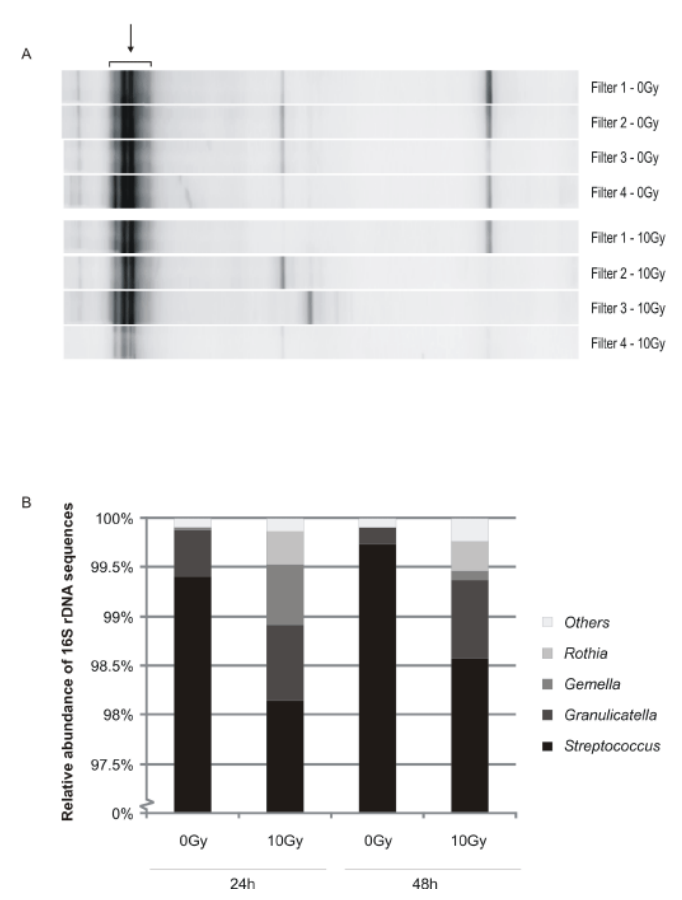

Figure 3: Effects of irradiation on the microbial composition. A: DGGE profiling of the oral microbiota present in the apical compartment after $24 \mathrm{~h}$ of co-culture with TR146 epithelial cells in irradiated (10 Gy) or non-irradiated (0 Gy) conditions. Indicated (arrow) is a group of GC-poor bacteria present in all samples $(\mathrm{n}=24)$ B: Pyrosequencing analysis of the oral microbiota cultured for 24 or $48 \mathrm{~h}$ in co-culture with TR146 epithelial cells in irradiated or non-irradiated conditions. Results of 454 pyrosequencing of $16 \mathrm{~S}$ rDNA assigned to genus are shown.

In summary, our study shows that irradiation can affect the hostmicrobe crosstalk during wound healing, depending on the composition and functionality of the microbiota. A slight, although not significant, increase in abundance of Rothia after irradiation could be noticed in our in vitro system, which might be correlated with mucositis-related local and/or systemic infections observed in vivo. 
Citation: Ryck TD, Boterberg T, Kerckhof FM, Schrijver JD, Bracke M, et al. (2015) Effects of Irradiation on Epithelial Wound Healing and Microbial Diversity in an in-vitro Oral Mucosa Model. J Nucl Med Radiat Ther 6: 218. doi:10.4172/2155-9619.1000218

Page 4 of 4

Further research is necessary to determine which species are likely to have a negative impact on the recovery of the epithelium after irradiation and to develop new strategies to combat infections associated with radiotherapy-induced mucositis.

\section{Acknowledgements}

We want to thank Karlien Van Wesemael for routinely checking the epithelial cells for mycoplasma contamination. Tine De Ryck was financially supported by an FWO project grant (G.0712.10 N). Tom Van de Wiele received funding an FWO research grant $(1426012 \mathrm{~N})$ and the GOA project (BOF12/GOA/008). Barbara Vanhoecke's research leading to these results has received funding from the Seventh Framework Programme (FP7/2011) under grant agreement n 299169 (Mucositis Platform). Frederiek-Maarten Kerckhof received funding of BOF (BOF09/GOA/005).

\section{References}

1. Elting LS, Cooksley CD, Chambers MS, Garden AS (2007) Risk outcomes, and costs of radiation-induced oral mucositis among patients with head-and-neck malignancies. Int J Radiat Oncol Biol Phys 68: 1110-1120.

2. Sonis ST (2007) Pathobiology of oral mucositis: novel insights and opportunities. J Support Oncol 5: 3-11.

3. Sonis ST (2004) The pathobiology of mucositis. Nat Rev Cancer 4: 277-284.

4. Wygoda a, Skladowski K, Rutkowski T, Hutnik M, Golen M, et al. (2012) Acute mucosal radiation reactions in patients with head and neck cancer.
Patterns of mucosal healing on the basis of daily examinations. Strahlenther Onkol 188: 686-691.

5. Bik EM, Long CD, Armitage GC, Loomer P, Emerson J, et al. (2010) Bacterial diversity in the oral cavity of 10 healthy individuals. ISME J 4: 962-974.

6. Vanhoecke B, De Ryck T, Stringer A, Van de Wiele T, Keefe D (2015) Microbiota and their role in the pathogenesis of oral mucositis. Oral Dis 21: 17-30.

7. De Ryck T, Grootaert C, Jaspaert L, Kerckhof FM, Van Gele M, et al. (2014) Development of an oral mucosa model to study host-microbiome interactions during wound healing. Appl Microbiol Biotechnol 98: 6831-6846.

8. Aguirre JS, Rodríguez MR, García de Fernando GD (2011) Effects of electron beam irradiation on the variability in survivor number and duration of lag phase of four food-borne organisms. Int J Food Microbiol 149: 236-246.

9. Aas JA, Paster BJ, Stokes LN, Olsen I, Dewhirst FE (2005) Defining the normal bacterial flora of the oral cavity. J Clin Microbiol 43: 5721-5732.

10. Hu YJ, Shao ZY, Wang Q, Jiang YT, Ma R, et al. (2013) Exploring the dynamic core microbiome of plaque microbiota during head-and-neck radiotherapy using pyrosequencing. PLoS One 8: e56343.

11. Hu YJ, Wang Q, Jiang YT, Ma R, Xia WW, et al. (2013) Characterization of oral bacterial diversity of irradiated patients by high-throughput sequencing. Int J Oral Sci 5: 21-25.

12. Ramanan P, Barreto JN, Osmon DR, Tosh PK (2014) Rothia bacteremia: a 10-year experience at Mayo Clinic, Rochester, Minnesota. J Clin Microbiol 52: 3184-3189. 